\title{
Endovascular repair by customized branched stent-graft: A promising treatment for chronic aortic dissection involving the arch branches
}

\author{
Qingsheng Lu, MD, ${ }^{\mathrm{a}}$ Jiaxuan Feng, MD, ${ }^{\mathrm{a}}$ Jian Zhou, MD, ${ }^{\mathrm{b}}$ Zhiqing Zhao, MD, ${ }^{\mathrm{b}}$ Haiyan Li, BSc,
}

Zhongzhao Teng, $\mathrm{PhD},{ }^{\mathrm{c}}$ and Zaiping Jing, $\mathrm{MD}, \mathrm{PhD}^{\mathrm{a}}$

\section{ABSTRACT}

Objective: There is no approved special endovascular device for use in preventing entry tears in the distal part of ascending aorta or in the aortic arch and preserving the arch branch arteries. Thus, we have designed a novel branched stent-graft, and herein report the initial clinical outcomes.

Methods: Between August 2009 and January 2014, 51 patients with aortic dissections involving the aortic arch were treated by endovascular branched stent-grafts. There were 7 Stanford type A aortic dissections, 22 retrograde type A aortic dissections, and 22 Stanford type B aortic dissections (including 4 localized aortic arch dissections). All patients were treated while in the chronic phase ( $>2$ weeks).

Results: All of the proximal entry tears in the arch were successfully excluded, and all of the treated branch arteries remained patent. No new cerebral infarction occurred. There was 1 death from a retrograde type A dissection, occurring 6 days after the endovascular procedure. The median follow-up period was 44 months (range, 14-66 months). No additional complications or mortality occurred. Complete thrombosis in the false lumen of the aortic arch was formed in all patients, and significant true lumen recovery and false lumen shrinkage were demonstrated in different levels of the thoracic aorta according to computed tomography angiography at 1 year postsurgery $(P<.001)$.

Conclusions: In patients with aortic dissection involving the arch branches, the customized branched stent-graft may provide a feasible endovascular treatment option. A larger series of cases with longer follow-up is needed to substantiate these results. (J Thorac Cardiovasc Surg 2015;150:1631-8)

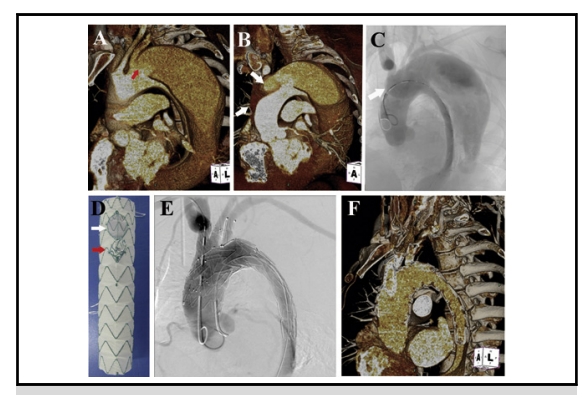

Aortic dissection involving the whole arch was treated with an endovascular branched stent-graft.

\section{Central Message}

The branched stent-graft has been subjected to 5 years of initial clinical evaluation with promising outcomes, as presented here. Customizing the branched stent-graft according to individual pathologies may provide a safe and effective endovascular treatment for patients with aortic dissections involving the arch branches.

\section{Perspective}

For patients with aortic dissection involving the arch branches that cannot be well managed by current endovascclar devices, this customized branched stent-graft may provide a safe and effective endovascular treatment.

See Editorial Commentary page 1639.

\footnotetext{
From the Departments of ${ }^{\mathrm{a}}$ Vascular Surgery and ${ }^{\mathrm{b}}$ Surgery, Changhai Hospital, Second Military Medical University, Shanghai, China and ${ }^{c}$ Department of Radiology, University of Cambridge, Cambridge, United Kingdom.

This work was supported by Grants from the National Natural Science Foundation of China $(81330034,81270386$, and 81273522) and the 1255 Project of Changhai Hospital (CH125520200 and CH125550300).

Drs Lu, Feng, and Zhou contributed equally to this work.

Received for publication May 14, 2015; revisions received July 30, 2015; accepted for publication Aug 11, 2015; available ahead of print Sept 16, 2015.

Address for reprints: Zaiping Jing, MD, PhD, Department of Vascular Surgery, Changhai Hospital, Yangpu District, Shanghai 200433, China (E-mail: xueguanky@163.com).

0022-5223

Copyright (C) 2015 by The American Association for Thoracic Surgery. Published by Elsevier Inc. This is an open access article under the CC BY-NC-ND license (http:// creativecommons.org/licenses/by-nc-nd/4.0/).

http://dx.doi.org/10.1016/j.jtcvs.2015.08.032
}

ß Supplemental material is available online.

Video clip is available online.

Aortic dissections involving the arch branches include dissections with entry tears located in the ascending aorta (Stanford type A aortic dissection), in the arch, or in the descending aorta with retrograde extension of the dissection 


\section{Abbreviations and Acronyms \\ CTA = Computed tomography angiography \\ IA $=$ Innominate artery \\ LCA $=$ Left common carotid artery \\ LSA $=$ Left subclavian artery \\ RCA $=$ Right common carotid artery \\ RTAD $=$ Retrograde type A aortic dissections}

into the ascending aorta (retrograde type A aortic dissection [RTAD]) and arch. The Stanford type A aortic dissections with arch involvement are treated mainly by open surgery with arch replacement in the acute phase. ${ }^{1,2}$ Kato and colleagues $^{3}$ developed a hybrid technique to improve open arch surgery, in which the stent-graft is deployed through the transected proximal aortic arch and arch replacement is performed with an artificial graft. This hybrid technique was later improved by a simplified procedure using a branched stentgraft inserted into the aortic arch and arch branches at the time of open surgery. ${ }^{4,5}$ These techniques continued to require median sternotomy and cardiopulmonary bypass, however, and were not well tolerated by high-risk patients. Thus, there was a need for endovascular interventions for this subgroup of patients.

Recent progress in endovascular treatment raised the idea that the use of stent-grafts might be an appropriate treatment option for aortic dissection with an entry tear located in the middle part of the ascending aorta. ${ }^{6}$ However, aortic dissection with an entry tear in the distal part of the ascending aorta (zone 0) still could not be treated by endovascular procedures; thus, there was a need for a specially designed endograft. ${ }^{7}$

Previous studies have reported that chimney and fenestration techniques may be feasible for treating some aortic dissections with entry tears in the arch or in the descending aorta (zones 1-3) with retrograde extension of the dissection into the arch and ascending aorta. ${ }^{8,9}$ In many cases, however, the distance between the arch branch arteries cannot provide a sufficient proximal landing zone. Thus, there was a need for hybrid techniques, including various methods to disconnect the arch branch arteries and transpose them to neighboring arteries or revascularize with bypasses. ${ }^{7}$ We asked whether we could develop a novel endovascular stent-graft so that the transthoracic or cervical hybrid operation could be avoided, or at least the transthoracic operation could be replaced by a cervical bypass procedure. Based on our previous studies, ${ }^{6,10-12}$ we have designed a 1-piece branched stent-graft system and have demonstrated its safe use in animal models. ${ }^{13,14}$ The purpose of the present study was to evaluate the feasibility of this branched stentgraft in treating aortic dissections involving the aortic arch.

\section{MATERIALS AND METHODS Patients}

This is a single-center retrospective study of a prospective database. Between August 2009 and January 2014, 51 consecutive patients with aortic dissections involving the aortic arch were treated using the branched stentgraft. The study group included 43 males $(84.3 \%)$ and 8 females $(15.7 \%)$, with a mean age of $65.5 \pm 6.9$ years (range, $56-79$ years). All patients were treated medically at the hospital at which they initially presented. The major indications for referral included refractory hypertension, persistent or recurrent chest pain and/or back pain, cerebrovascular events (ie, transient ischemia attack or stroke), progressive dilatation of the aorta, pleural effusion, and compression symptoms, such as hoarseness because of recurrent laryngeal nerve compression by an aneurysmal false lumen.

The study cohort included 7 patients with aortic dissection with the entry tear located in the distal part of the ascending aorta (zone 0). A multidisciplinary consultation of vascular surgeons, cardiac surgeons, cardiologists, and anesthetists was provided to these patients, who were deemed at high risk for open surgical repair because of age $>70$ years $(\mathrm{n}=5)$ or history of median sternotomy and previous aortic surgery $(\mathrm{n}=2)$, combined with other comorbid conditions (Table E1). The remaining 44 patients $(86.3 \%)$ had proximal entry tears located in the arch or conjunction part of the aortic arch and descending aorta (zones 1-3), with retrograde involvement of the ascending aorta in 22 patients, retrograde involvement of the aortic arch in 18 patients, and localized involvement of the aortic arch in 4 patients. Six proximal entry tears were located in zone 1,15 entry tears were located in zone 2, and 23 entry tears were located in zone 3 . Characteristics of the aortic dissections are presented in Table E2. The anatomy of the arch and pathologies excluded these patients from treatment using chimney techniques. The patients refused hybrid procedures that required transthoracic bypass and agreed to endovascular treatment with the branched stent-graft.

Patients with following conditions were considered unfit for the endovascular branched stent-graft: aortic dissection involving coronary artery orifice or aortic valve, which may cause severe aortic regurgitation (grade 3 or 4 , documented by echocardiography); history of potentially life-threatening ventricular arrhythmia; severe tortuosity or narrowing of the access artery (including the descending aorta, abdominal aorta, and iliac artery); or a connective tissue disorders (eg, Marfan syndrome, Ehlers-Danlos syndrome). All of the enrolled patients were informed about the experimental characteristics of the endovascular branched stent-graft, and all provided informed consent. Initial application of this branched stent-graft in humans was approved by the Institutional Review Board of Changhai Hospital.

\section{Stent-Graft Design}

The location of the entry tears, anatomic features of the aortic arches, and the direction of the supra-arch arteries were determined using 3-dimensional vascular images (Figure 1). Based on these measurements, we made draft drawings of the branched stent-graft, which is designed to seal the entry tear with a sufficient landing zone and preserve arch branch arteries using branches and fenestrations. Cervical bypass and intentional occlusion of arch branch arteries were avoided wherever possible. A fenestration close to the entry tear (distance $<10 \mathrm{~mm}$ ) was considered to represent a high risk of endoleak. In this situation, the branch artery was reconstructed with a branch section of the stent-graft, or by cervical bypass if the branch section had to be placed into another arch vessel. Stable patients awaiting such customization were discharged and treated medically.

The branched stent-grafts were manufactured by MicroPort Medical Co, Ltd (Shanghai, China) (Figure 1). Previous ex vivo endograft tests and animal experiments were performed under the supervision of the State Food and Drug Administration of China. Branched stent-grafts were specifically designed for each individual patient (Table 1). 

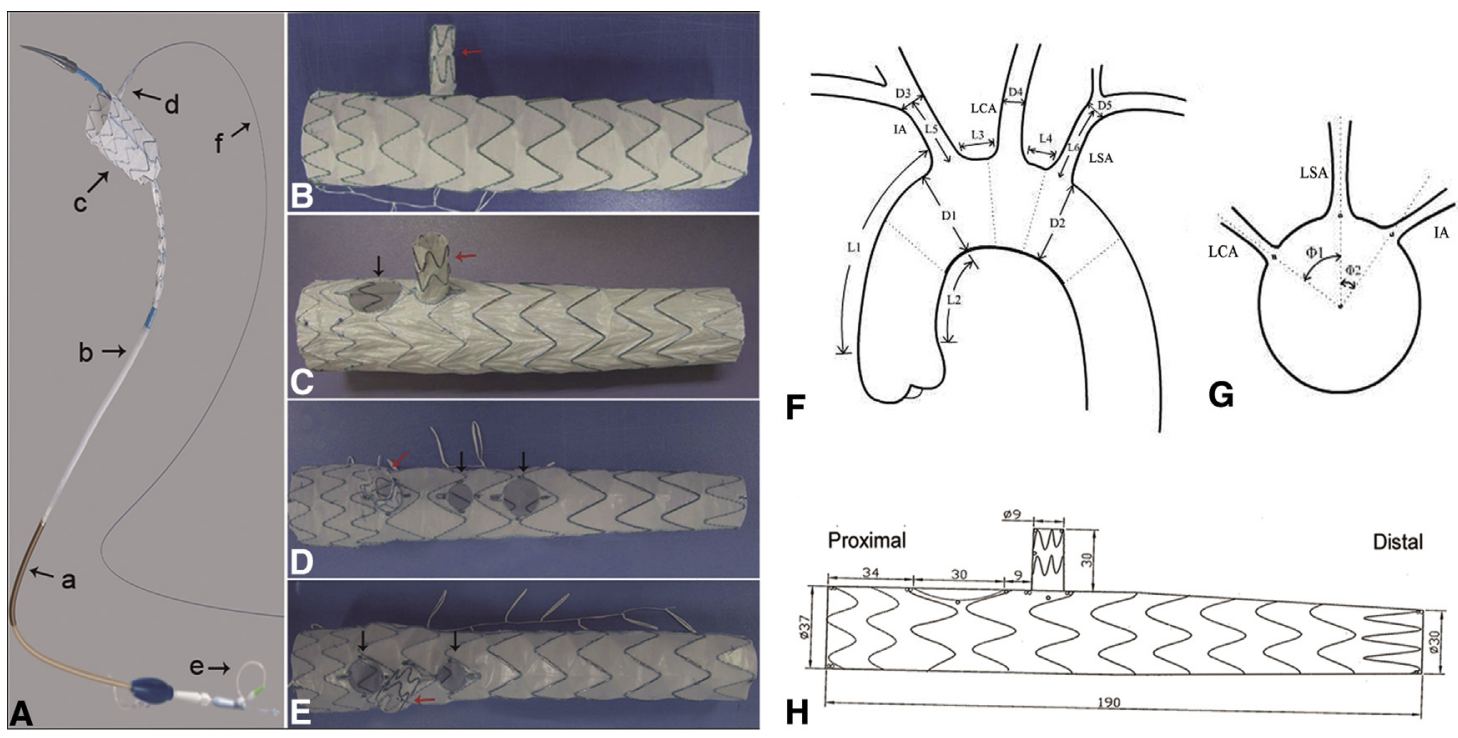

FIGURE 1. Construction of the customized 1-piece branched stent-graft. A, The configuration of the branched stent-graft and delivery system. The branched stent-graft is constructed of woven polyester fabric sewn to self-expanding Nitinol stents, without a proximal or distal bare stent. The delivery system consists of an outer $22 \mathrm{Fr}$ sheath with a low-friction hydrophilic coating on the outer sheath (a) and an inner soft polyester fabric sheath (b). The soft sheath encapsulates the aortic graft trunk (c) and the branch section (d), which are folded individually. The aortic graft trunk is folded with loops of thread and a nickel titanium wire (trigger wire) (e), and the branch section is contained by a cap, constructed from polyester fabric and attached to a traction wire (f). In this way, the 2 sections can be deployed consecutively rather than simultaneously. A steel ring is sewn to the origin of the branch section to keep this branch section open and prevent stenosis, which may occur in the process of aortic remodeling. B, Branched stent-graft without fenestration. C, Branched stent-graft with proximal single fenestration. D, Branched stent-graft with double distal fenestration. E, Branched stent-graft with 1 proximal fenestration and 1 distal fenestration. The red arrows indicate the branch section, and the black arrows show the fenestrations. F, Measuring the aortic arch on 3-dimensional vascular images reconstructed from the aortic CTA on a TeraRecon Aquarius workstation (TeraRecon, Foster City, Cal). The diameter and longitudinal distance of the arch are measured to determine the proper size of the stent-graft, as well as the distance between the fenestration and the branch section of the stent-graft. G, Measuring the circumferential angles of the arch branch arteries on the axial vision of the aorta, to determine the angles between the fenestration and the branch section on the stent-graft. H, A sketch used for customizing the branched stent-graft with single proximal fenestration, to clarify which measurements are important. There are 4 radioactive marks (eg, "8," "0") around the fenestration or the root of the branch section. LCA, Left common carotid artery; $L S A$, left subclavian artery; $I A$, innominate artery.

\section{Endovascular Procedure}

General anesthesia was administered in all cases. If the intended artery of the branch section of the stent-graft was the innominate artery (IA) or left subclavian artery (LSA), then the right or left brachial artery ("traction artery") was exposed and cannulated with a short $8 \mathrm{Fr}$ sheath. This traction artery was used to draw the branch section of the stent-graft into its designated artery. If the branch section was intended for the left common carotid artery (LCA), then the traction artery could be the left brachial artery if an LCA-LSA bypass had been performed previously, because the guidewire could navigate through the bypass from the LSA to the LCA (eg, in patient 12). If there was no previous LCA-LSA bypass, then the LCA served as the traction artery and was exposed through a cervical incision (eg, in patient 19). Through the sheath in the traction artery, a 4 Fr catheter was advanced over a guidewire to the femoral artery and was taken out from the arteriotomy site (Figure 2, $A$ ). The guidewire was then withdrawn, leaving the $4 \mathrm{Fr}$ catheter as the traction conduit, through which the traction wire of the branch section of the stent-graft exited through the sheath in the traction artery.

The branched stent-graft was advanced into the descending aorta over the super-stiff guidewire (Figure 2, B). With the outer sheath remaining in the descending aorta, the stent-graft within the soft sheath was advanced into the arch (Figure 2,C). While delivering the stent-graft from the femoral arteriotomy site to the arch, it was important to ensure that the branch section of stent-graft followed the greater curvature of the aortic arch. Rotating the stent-graft in the transverse arch was difficult and risky. If the branch section was not on the correct side of the arch, or if the traction wire became twisted around the stent-graft, then the stent-graft could be withdrawn into the outer sheath in the descending aorta, rotated to the correct direction, and readvanced into the arch.

Once the stent-graft was delivered to the planned location in the arch, the soft sheath was removed. Then the branch section inside its cap could be pulled into the intended branch artery by drawing the traction wire (Figure 2,D). The stent-graft trunk was quickly deployed by withdrawing the trigger wire (Figure 2,E). Only after removal of the trigger wire was the cap of the branch section unlocked and removed by withdrawing the traction wire, which deployed the branch section (Figure 2, F). Immediate aortography identified whether the entry tear was excluded and vital branch arteries were patent. Video 1 shows the endovascular procedure using the branched stent-graft in patient 25 .

\section{Follow-up Examinations}

Patients were followed with computed tomography angiography (CTA) of the aorta, color Doppler ultrasound of the cervical arteries, and cerebral magnetic resonance imaging at 6-month intervals during the first year postsurgery, and then annually thereafter. Three-dimensional vascular images reconstructed from CTA at 1 year were used to evaluate false-lumen thrombosis and aortic remodeling. Morphological remodeling was measured at the orifice of the IA (level A), the orifice of the LCA (level B), the orifice 
TABLE 1. Configuration parameters of the branched stent-graft

\begin{tabular}{lc}
\hline \multicolumn{1}{c}{ Parameter } & Value \\
\hline Proximal aortic trunk diameter, mm & $28-40$ \\
Distal aortic trunk diameter, mm & $25-38$ \\
Branch section diameter, mm & $7.5-14$ \\
Oversize rate of proximal landing zone, \% & $7.4 \pm 5.0$ \\
Oversize rate of distal landing zone, $\%$ & $5.9 \pm 5.4$ \\
Oversize rate of the branch section, $\%$ & $5.7 \pm 2.0$ \\
Tapered stent-grafts, n ( $\%$ ) & $46(90.2)$ \\
Taper ratio of the tapered stent-grafts, $\%$ & $17.8 \pm 11.5$ \\
Length of the proximal landing zone, $\%$ & $38.2 \pm 11.1$ \\
Length of the distal landing zone, $\%$ & $88.6 \pm 36.0$ \\
Length of the branch section, mm & IA: $26.2 \pm 5.2$ \\
& LCA: $20.3 \pm 4.5$ \\
& LSA: $25.2 \pm 5.6$ \\
\hline
\end{tabular}

The proximal part of the stent-graft was landed in the segment of ascending aorta or aortic arch that was not involved in the dissection. The outer diameter of the entire aortic lumen was measured as the diameter of the proximal landing zone. The oversize rate was defined as (stent-graft diameter - target artery diameter)/(target artery diameter $) \times 100 \%$. Fifty-one stent-grafts $(92.6 \%)$ were tapered to fit the changing diameter from the ascending aorta to the arch to the descending aorta. The taper ratio of the stent-graft was defined as (proximal diameter - distal diameter)/(proximal diameter $) \times 100 \%$. The length of the proximal landing zone was measured from the proximal edge of the trunk to the first fenestration or branch section. The length of the distal landing zone was measured from the distal edge of the trunk to the last fenestration or branch. IA, Innominate artery; $L C A$, left common carotid artery; $L S A$, left subclavian artery.

of LSA (level C), and the distal end of the stent-graft (level D) (Figure 2, F). The maximal diameter of the aortic dissection was measured as well.

\section{Statistical Analysis}

Continuous variables were recorded as mean \pm standard deviation, and skewed variables were recorded as median and range. Diameters were compared using a 2-sided paired-sample $t$ test. SPSS version 13.0 (SPSS Inc, Chicago, Ill) was used for all analyses. A $P$ value $<.05$ was considered significant.

\section{RESULTS}

The interval from acute dissection to branched stentgrafting procedure ranged from 31 days to 72 days (median, 39 days), except in the extreme case of patient 7 . Thus, all patients were treated while in the chronic phase ( $>2$ weeks). Patient 7 was referred to our center in the 35 th month owing to a significant expansion of a false lumen and underwent endovascular intervention on the 1080th day after aortic dissection occurred. The time for customizing the branched stent-graft ranged from 18 days to 43 days (median, 22 days). No patient died or suffered major complications while awaiting customization.

\section{Configuration of the Branched Stent-Grafts}

A total of 53 branched stent-grafts were used in this study. The individual arch-reconstructing strategies are presented in Table E2. Four types of branched stent-graft were designed in this study.

An overlapped 2 single-branched stent-graft was used in 2 patients (patients 1 and 14). In these patients, 2 single-branched stent-grafts were overlapped for at least $25 \mathrm{~mm}$ (Figure E3). One branch section was placed into the IA, and the other was placed into the LSA. The LCA was reconstructed by a right common carotid artery (RCA)-LCA bypass.

A single-branched stent-graft was used in 22 patients (Figure 1, B). The proximal landing zone was in zone 2 in 18 of these patients whose LSA was preserved by branch sections of the stent-graft, and in zone 0 in 4 patients whose IA was preserved by branch sections, with the LCA and LSA reconstructed by cervical bypass (patients $7,8,13$, and 34).

A single-branched stent-graft with 1 fenestration was used in 17 patients (Figure 1,C). In 13 of these patients, branch sections of the stent-graft were placed into the LSA, and the fenestration was aligned with the origins of the other arch branch arteries. Among these 13 patients, the fenestration aligned with the 2 origins of the IA and LCA in 11 patients (Figure 3) and with the origin of IA in 2 patients, in whom carotid-carotid bypass was performed to reconstruct the LCA. In 3 patients, branch sections were placed into the LCA, fenestrations were aligned with the IA, and LCA-LSA bypass was done to reconstruct the LSA. In 1 patient (patient 2), who had aberrant arch branch arteries as described above, the branch section was placed into the right subclavian artery, and the remaining arch branches were preserved by 1 fenestration.

Finally, 10 patients were treated with a single-branched stent-graft with 2 fenestrations (Figure $1, D$ and $E$ ). In this group were 5 patients treated by a branched stentgraft with double proximal fenestrations, 3 patients treated by a branched stent-graft with double distal fenestrations, and 2 patients treated by a branched stent-graft with 1 proximal fenestration and 1 distal fenestration.

In total, there were 53 branch sections and 37 fenestrations on the stent-grafts. Six of the 37 fenestrations were stented $(16.2 \%)$. Eleven cervical bypasses were performed in this cohort (21.6\%), and no complications related to the bypass procedures were observed. Thirty-three proximal landing zones were located in zone $0(64.7 \%)$, and 18 were in zone $2(35.3 \%)$.

\section{Perioperative Outcomes}

The mean duration of the procedure was $134.7 \pm 26.9$ minutes. All of the branched stent-grafts were deployed at the planned locations. All of the arch branch arteries were patent, and no type I/III endoleaks were detected on completion aortography. None of the patients was admitted to the intensive care unit, and there were no cases of in-hospital stroke. One death occurred in the postoperative period, in patient 5 , a female with a proximal entry tear in zone 1 treated by a branched stent-graft with a single proximal fenestration. The branch section was placed into the LSA, the fenestration was 

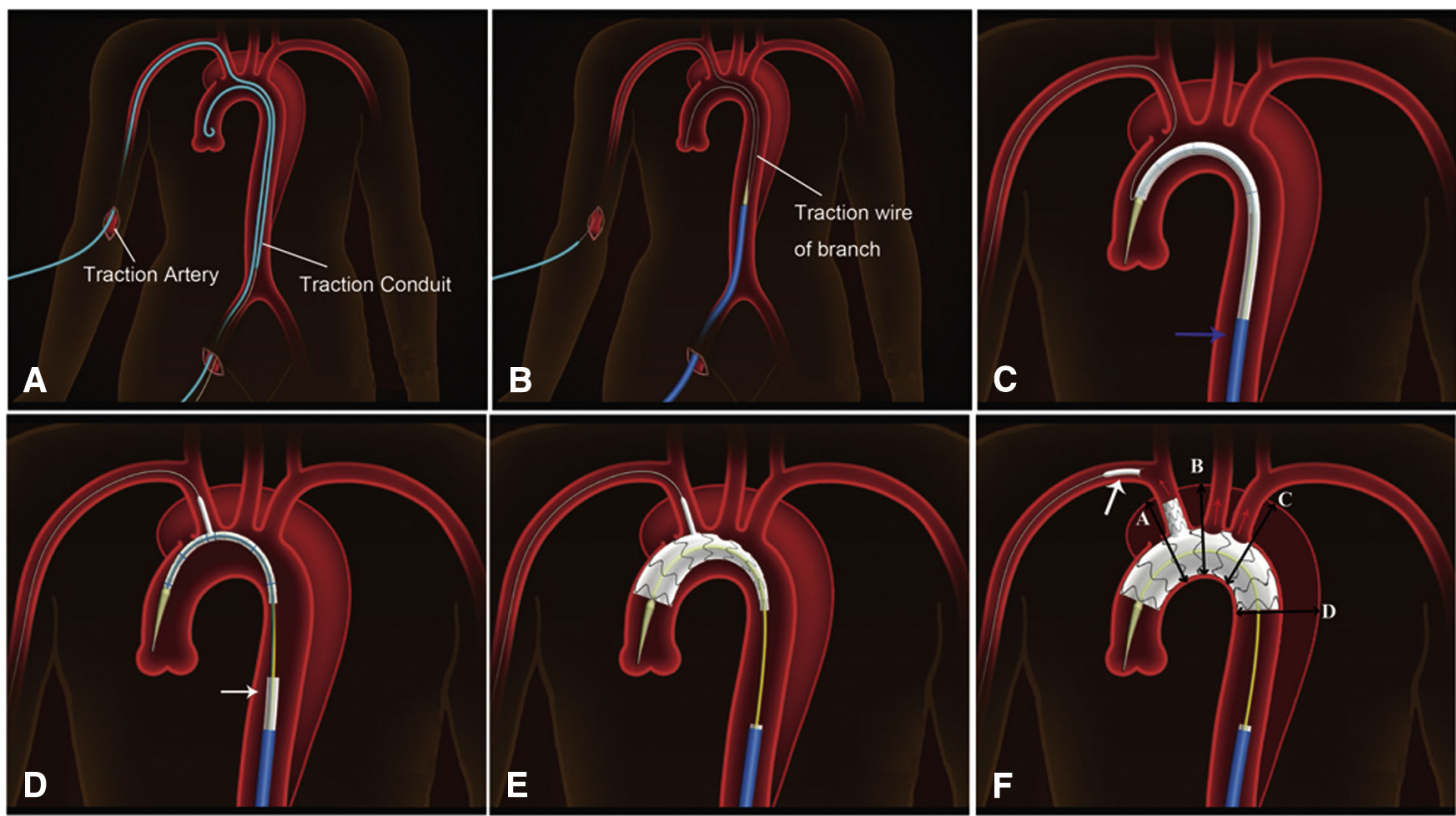

FIGURE 2. Still images of the movie of endovascular procedure using this branched stent-graft (using the procedure for patient 25 as an example). A, Establishing the traction conduit. B, Advancing the branched stent-graft into the descending aorta, with synchronous withdrawal of the traction conduit and traction wire of the branch section. C, Advancing the stent-graft to the ideal position in the arch. The outer sheath remains in the descending aorta (blue arrow). D, Drawing the branch section into the intended branch artery. The white arrow shows that the soft sheath of the stent-graft has been removed. E, Deploying the main body of the stent-graft. F, Deploying the branch section. The white arrow indicates the removed cap. The diameters of the aorta, true lumen, and false lumen at levels A-D were measured at the 1-year follow-up in all patients.

aligned with the IA, and the LCA was reconstructed by an RCA-LCA bypass. Her final angiography showed that the proximal entry tear was completely excluded and the arch branch arteries were successfully preserved. She complained of chest pain at 6 days after the procedure, and emergent CTA revealed an RTAD with entry tear located in the larger curve side of the proximal edge of the stentgraft. She died suddenly during preparation for surgery. The 30-day outcomes are presented in Table E3.

\section{Follow-up and Morphological Remodeling}

No patients were lost to follow-up. The median follow-up period was 44 months (range, 14-66 months). On follow-up, all of the arch branch arteries and cervical bypasses were patent, no fenestration lost its alignment, and no branch section of the stent-graft was kinked or occluded. No follow-up complications or mortality were noted. All patients exhibited complete thrombosis of the false lumen in the ascending aorta and arch at the last CTA examination. In the descending aorta, complete thrombosis of the false lumen was noted in 47 of the 51 patients $(92.2 \%)$, and partial thrombosis was noted in the other 4 patients $(7.8 \%)$ because of the flow coming from distal reentry tears.

Regarding aortic remodeling at the 1-year follow-up, significant morphological changes were noted at 4 designated levels (Table 2); diameters of the whole aorta and false lumens at the 4 levels were significantly decreased
$(P<.001)$, and the true lumens were significantly expanded $(P<.001)$ (Figure E3, $H$ and $I)$. The maximal diameters of the dissected aorta were significantly decreased at 1 year after endovascular repair $(P<.001)$.

\section{DISCUSSION}

The continued development of endovascular stent-grafts has allowed their use in the proximal descending aorta. Attention has recently focused on the ascending aorta and aortic arch. ${ }^{6}$ Chimney techniques and fenestrated devices have been used to treat some selected patients with aortic arch pathologies. ${ }^{8,10,11,15,16}$ For chimney techniques, the incidence of intraoperative endoleaks and the risk of cerebral infarction remain under evaluation. ${ }^{17}$ In some cases, the anatomy of the aortic arch and location of the entry tear carry a high risk of endoleak in the endovascular procedure using a chimney technique; examples include entry tears located very close to the origin of the arch branch arteries and overly short distances between the arch branches.

When using fenestrated devices, aligning the fenestration with the origin of the branch arteries in the aortic arch is more difficult and risky than doing so in the visceral part of abdominal aorta because of the curve of the aortic arch. A typical all-fenestrated endograft is the Najuta device. ${ }^{18}$ Unexpected or intentional coverage of the arch branch arteries is not a rare occurrence, according to a recent report. ${ }^{16}$ This situation has led to the development 

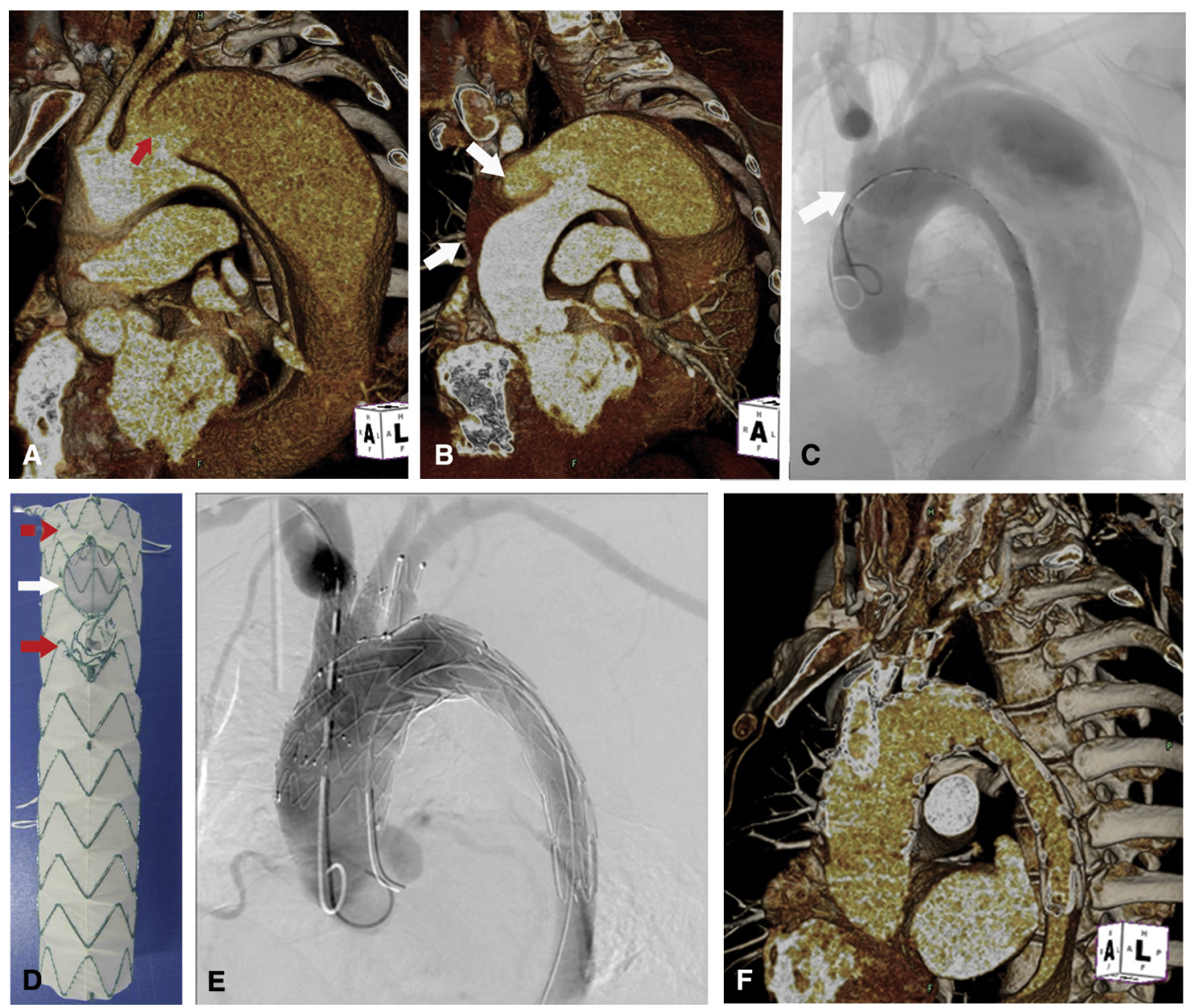

FIGURE 3. Endovascular treatment of aortic dissection affecting the entire aortic arch by branched stent-graft with 1 fenestration. A, In patient 6 , the proximal entry tear located in zone 2, at the same level as the origin of the LSA (red arrow). B, Retrograde extension of the aortic dissection into the arch and ascending aorta (white arrow). C, Aortography showing a retrograde type A aortic dissection. The false lumen was significantly expanded, and the true lumen was severely narrowed. D, A branched stent-graft with proximal fenestration. The red arrow indicates the branch section, and the white arrow points to the fenestration on the stent-graft. E, Final aortography showing the IA and LCA sharing the same origin in the arch, which was preserved by a single fenestration. The LSA was reconstructed with the branch section. The coronary arteries were patent. F, Four-year follow-up CTA revealing patency of the supra-arch branches and complete thrombosis of the false lumen.

of in situ fenestration techniques using needle, laser, or radiofrequency punctures ${ }^{9}$; however, the technical success rate of these procedures remains questionable. Moreover, the possibility of loss of alignment during follow-up warrants rigorous follow-up. ${ }^{19}$ Our combination of a fenestration technique with a branched stent-graft not only can address more situations of complicated aortic arch pathologies, but also can control the risk of secondary displacement of fenestrations during follow-up, owing to the anchoring effect of the branch section on the stent-graft.

The idea of using an endovascular branched stent-graft in the aortic arch is not totally new. Chuter and colleagues ${ }^{20}$ developed a modular branched stent-graft to preserve the IA, followed by cervical bypass. This design was further modified and tested in animal models ${ }^{21}$; however, it sacrificed the LSA, a controversial approach that has not been justified in the clinical setting. ${ }^{22}$ Inoue and colleagues $^{23}$ designed a 1-piece branched stent-graft to treat distal arch pathologies involving the LSA, but found a relatively high rate of complications, including endoleak, cerebral infarction, and access site complications. Although additional data from that group suggested the feasibility of this branched stent-graft in treating aortic pathologies involving the LSA, the type I endoleak rate in aortic dissection was found to be as high as $28.6 \% .^{24}$ The relatively weak sealing ability of the nickel titanium rings, along with the short landing zone between the origins of the LSA and LCA, might contribute to this high endoleak rate. $^{25}$

In our cohort, if the ascending aorta or the proximal part of aortic arch was affected by the aortic dissection, then the proximal landing zone was extended into the ascending 
TABLE 2. Morphological changes along the aortic arch at 1 year after branched stent-graft implantation

\begin{tabular}{lccc}
\hline \multicolumn{1}{c}{ Level } & $\begin{array}{c}\text { Before } \\
\text { endografting, } \mathbf{~ m m}\end{array}$ & $\begin{array}{c}\text { 1-year } \\
\text { follow-up, } \mathbf{~ m m}\end{array}$ & $\begin{array}{c}\boldsymbol{P} \\
\text { value }\end{array}$ \\
\hline Level A & & & \\
$\quad$ Aorta & $42.6 \pm 3.7$ & $35.3 \pm 2.9$ & $<.001$ \\
True lumen & $27.0 \pm 5.3$ & $32.7 \pm 2.7$ & $<.001$ \\
False lumen & $13.9 \pm 8.0$ & $4.6 \pm 3.3$ & $<.001$ \\
Level B & & & \\
Aorta & $39.3 \pm 3.4$ & $34.9 \pm 3.3$ & $<.001$ \\
True lumen & $25.5 \pm 3.3$ & $30.0 \pm 2.8$ & $<.001$ \\
False lumen & $12.5 \pm 6.2$ & $4.4 \pm 3.0$ & $<.001$ \\
Level C & & & \\
Aorta & $37.3 \pm 3.6$ & $34.6 \pm 3.1$ & $<.001$ \\
True lumen & $21.0 \pm 2.8$ & $28.6 \pm 2.0$ & $<.001$ \\
False lumen & $15.2 \pm 4.0$ & $4.9 \pm 2.4$ & $<.001$ \\
Level D & & & \\
Aorta & $39.6 \pm 6.3$ & $29.4 \pm 4.6$ & $<.001$ \\
$\quad$ True lumen & $13.6 \pm 10.2$ & $24.4 \pm 5.0$ & $<.001$ \\
False lumen & $24.2 \pm 7.5$ & $4.9 \pm 3.8$ & $<.001$ \\
Maximal dissected aorta & $57.9 \pm 9.9$ & $38.5 \pm 3.0$ & $<.001$ \\
\hline Values are mean \pm SD. & & &
\end{tabular}

aorta to provide a sufficient landing length. No type I endoleaks occurred. The risk of extending the proximal landing zone into the ascending aorta remains controversial. Data from a transcontinental registry showed an incidence of RTAD of up to $7.5 \%$ during zone 0 total debranching ${ }^{26}$; however, this increased risk may result from aortic side-clamping, which has been reported to increase the risk of aortic dissection. ${ }^{27}$ One study using the Medtronic Thoracic Endovascular Registry reported a $0 \%$ rate of RTAD after endovascular repair landing in zone $0 .{ }^{28} \mathrm{In}$ the present study, 1 RTAD occurred after endovascular repair $(3.0 \%)$ in which the proximal landing zone was in the curved section of zone 0 . A previous study suggested that extending the landing zone into the straight part of aorta and not the curved part might prevent stent-graft-related new dissection. ${ }^{29}$ Thus, after the aforementioned case, in cases where the proximal landing zone was planned in zone 0 , we designed a branched stent-graft with a longer proximal landing length (approximately $40 \mathrm{~mm}$ ) that could reach the straight part of the ascending aorta, and no further cases of RTAD occurred after endovascular repair.

Along with completely excluding the proximal entry tear and preventing aortic wall injury, major concerns with this procedure include maintaining adequate intraoperative cerebral perfusion and ensuring long-term patency of the arch branch arteries. Thus, the delivery system, the structure of this branched stent-graft, and the method of placing the branch section into the branch artery were designed to improve intraoperative manipulation.

The outer sheath does not enter the aortic arch, but remains in the descending aorta during the procedure. The stent-graft can be retrieved from the arch and placed back into the outer sheath when it needs to be rotated to the proper direction. In this way, manipulation in the aortic arch can be reduced, thereby reducing the risk of intimal injury. In addition, when the stent-graft is delivered to the transverse arch, the entire endograft is covered by the soft inner sheath, which can protect the fragile aortic wall from the rough surface of the stent-graft. We believe that this reduces the risk of intimal injury and cerebral embolization. Moreover, the branch section of the stentgraft is enfolded by a cap made of the same fabric as the soft inner sheath. This design also helps prevent intimal injury while the branch section is drawn into the branch artery. The profile of the folded branch section is small enough ( $8 \mathrm{Fr}$ ) to be safely drawn into the branch arteries. In addition, the conjunction part of branch section and the aortic graft trunk is made of soft polyester fabric, allowing the branch section to be easily drawn into the branch artery.

The rapid and sequential deployment of the aortic graft trunk and the branch section prevents stent-graft twisting or malpositioning caused by the high-velocity pulsatile blood flow in the aortic arch. In addition, no more than 10 seconds elapse between the time of aortic trunk deployment and branch section deployment. Any interruption of cerebral perfusion due to a temporary branch block is so short as to not result in neurologic injury. Finally, when the branch section is drawn into the branch artery, the fenestrations align with the origins of the intended branch arteries, making aligning the fenestrations with the branch arteries much easier and safer than when using an allfenestrated stent-graft. This feature clearly relies on accurate preoperative measurement and good stent-graft design.

\section{Limitations}

Limitations of this study include the small sample size, short follow-up period, and lack of a control group. Future studies should compare the clinical results of this endovascular branched stent-graft with existing methods, such as open surgery, hybrid procedures, and chimney techniques. In addition, patients with aortic arch atherosclerotic aneurysms were not included in this study. The design principles of the branched endograft are different for these aneurysms, and should be evaluated in another study.

\section{CONCLUSIONS}

The results of this study of the use of a branched stent-graft to treat complicated aortic dissection involving the arch have given us an optimistic view of this novel endovascular device. Based on adequate preoperative planning and design, endovascular repair using this branched stent-graft may prove to be an acceptable alternative therapy for open surgery and hybrid procedures. Larger controlled studies with longer follow-up times are needed. 


\section{Conflict of Interest Statement}

Authors have nothing to disclose with regard to commercial support.

We thank Clifford J. Buckley, MD, Scott \& White Clinic in Temple, Texas, for valuable suggestions in preparing the manuscript.

\section{References}

1. Hagan PG, Nienaber CA, Isselbacher EM, Bruckman D, Karavite DJ, Russman PL, et al. The International Registry of Acute Aortic Dissection (IRAD): new insights into an old disease. JAMA. 2000;283:897-903.

2. Ochiai Y, Imoto Y, Sakamoto M, Ueno Y, Sano T, Baba H, et al. Long-term effectiveness of total arch replacement for type A aortic dissection. Ann Thorac Surg. 2005;80:1297-302.

3. Kato M, Ohnishi K, Kaneko M, Ueda T, Kishi D, Mizushima T, et al. New graftimplanting method for thoracic aortic aneurysm or dissection with a stented graft. Circulation. 1996;94(9 Suppl):II188-93.

4. Chen LW, Dai XF, Lu L, Zhang GC, Cao H. Extensive primary repair of the thoracic aorta in acute type A aortic dissection by means of ascending aorta replacement combined with open placement of triple-branched stent graft: early results. Circulation. 2010;122:1373-8.

5. Chen LW, Wu XJ, Lu L, Zhang GC, Yang GF, Yang ZW, et al. Total arch repair for acute type A aortic dissection with 2 modified techniques: open singlebranched stent graft placement and reinforcement of the dissected arch vessel stump with stent graft. Circulation. 2011;123:2536-41.

6. Lu Q, Feng J, Zhou J, Zhao Z, Bao J, Feng R, et al. Endovascular repair of ascending aortic dissection: a novel treatment option for patients judged unfit for direct surgical repair. J Am Coll Cardiol. 2013;61:1917-24.

7. Criado FJ, Barnatan MF, Rizk Y, Clark NS, Wang CF. Technical strategies to expand stent-graft applicability in the aortic arch and proximal descending thoracic aorta. J Endovasc Ther. 2002;9(Suppl 2):II32-8.

8. Xue Y, Sun L, Zheng J, Huang X, Guo X, Li T, et al. The chimney technique for preserving the left subclavian artery in thoracic endovascular aortic repair. Eur J Cardiothorac Surg. 2015;47:623-9.

9. Tse LW, Lindsay TF, Roche-Nagle G, Oreopoulos GD, Ouzounian M, Tan KT. Radiofrequency in situ fenestration for aortic arch vessels during thoracic endovascular repair. J Endovasc Ther. 2015;22:116-21.

10. Feng R, Zhao Z, Bao J, Wei X, Wang L, Jing Z. Double-chimney technology for treating secondary type I endoleak after endovascular repair for complicated thoracic aortic dissection. J Vasc Surg. 2011;54:212-5.

11. Yuan L, Feng X, Jing Z. Endovascular repair of a thoracic arch aneurysm with a fenestrated stent-graft. J Endovasc Ther. 2008;15:539-43.

12. Lu Q, Jing Z, Zhao Z, Bao J, Feng X, Feng R, et al. Endovascular stent graft repair of aortic dissection type B extending to the aortic arch. Eur J Vasc Endovasc Surg. 2011;42:456-63.

13. Lin C, Lu Q, Liao M, Guo M, Gong J, Jing Z. Endovascular repair of the half aortic arch in pigs with an improved, single-branched stent graft system for the brachiocephalic trunk. Vascular. 2011;19:242-9.
14. Lin C, Wang L, Lu Q, Li C, Jing Z. Endovascular repair of the aortic arch in pigs by improved double-branched stent grafts. Ann R Coll Surg Engl. 2013; 95:134-9.

15. Criado FJ. A percutaneous technique for preservation of arch branch patency during thoracic endovascular aortic repair (TEVAR): retrograde catheterization and stenting. J Endovasc Ther. 2007;14:54-8.

16. Azuma T, Yokoi Y, Yamazaki K. The next generation of fenestrated endografts: results of a clinical trial to support an expanded indication for aortic arch aneurysm treatment. Eur J Cardiothorac Surg. 2013;44:e156-63.

17. Moulakakis KG, Mylonas SN, Dalainas I, Sfyroeras GS, Markatis F, Kotsis T, et al. The chimney-graft technique for preserving supra-aortic branches: a review. Ann Cardiothorac Surg. 2013;2:339-46.

18. Kawaguchi S, Yokoi Y, Shimazaki T, Koide K, Matsumoto M, Shigematsu H. Thoracic endovascular aneurysm repair in Japan: experience with fenestrated stent grafts in the treatment of distal arch aneurysms. J Vasc Surg. 2008;48:24S-9S.

19. Scurr JR, How TV, McWilliams RG, Lane S, Gilling-Smith GL. Fenestrated stent-graft repair: which stent should be used to secure target vessel fenestrations? J Endovasc Ther. 2008;15:344-8.

20. Chuter TA, Schneider DB, Reilly LM, Lobo EP, Messina LM. Modular branched stent graft for endovascular repair of aortic arch aneurysm and dissection. $J$ Vasc Surg. 2003;38:859-63.

21. Wei G, Xin J, Yang D, Liu X, Tai Y, Zhang H, et al. A new modular stent graft to reconstruct aortic arch. Eur J Vasc Endovasc Surg. 2009;37:560-5.

22. Zhang L, Zhou J, Lu Q, Zhao Z, Bao J, Jing Z. Potential risk factors of reintervention after endovascular repair for type B aortic dissections. Catheter Cardiovasc Interv. 2015;86:E1-10.

23. Inoue K, Hosokawa H, Iwase T, Sato M, Yoshida Y, Ueno K, et al. Aortic arch reconstruction by transluminally placed endovascular branched stent graft. Circulation. 1999;100(19 Suppl):II316-21.

24. Saito N, Kimura T, Odashiro K, Toma M, Nobuyoshi M, Ueno K, et al. Feasibility of the Inoue single-branched stent-graft implantation for thoracic aortic aneurysm or dissection involving the left subclavian artery: short- to medium-term results in 17 patients. J Vasc Surg. 2005;41:206-12.

25. Schoder M, Grabenwöger M, Hölzenbein T, Cejna M, Ehrlich MP, Rand T, et al. Endovascular repair of the thoracic aorta necessitating anchoring of the stent graft across the arch vessels. J Thorac Cardiovasc Surg. 2006;131:380-7.

26. Czerny M, Weigang E, Sodeck G, Schmidli J, Antona C, Gelpi G, et al. Targeting landing zone 0 by total arch rerouting and TEVAR: midterm results of a transcontinental registry. Ann Thorac Surg. 2012;94:84-9.

27. Chavanon O, Carrier M, Cartier R, Hébert Y, Pellerin M, Pagé P, et al. Increased incidence of acute ascending aortic dissection with off-pump aortocoronary bypass surgery? Ann Thorac Surg. 2001;71:117-21.

28. Canaud L, Ozdemir BA, Patterson BO, Holt PJ, Loftus IM, Thompson MM. Retrograde aortic dissection after thoracic endovascular aortic repair. Ann Surg. 2014;260:389-95.

29. Dong Z, Fu W, Wang Y, Wang C, Yan Z, Guo D, et al. Stent graft-induced new entry after endovascular repair for Stanford type B aortic dissection. J Vasc Surg. 2010;52:1450-7.

Key Words: endovascular repair, aortic arch, aortic dissection, stent graft 

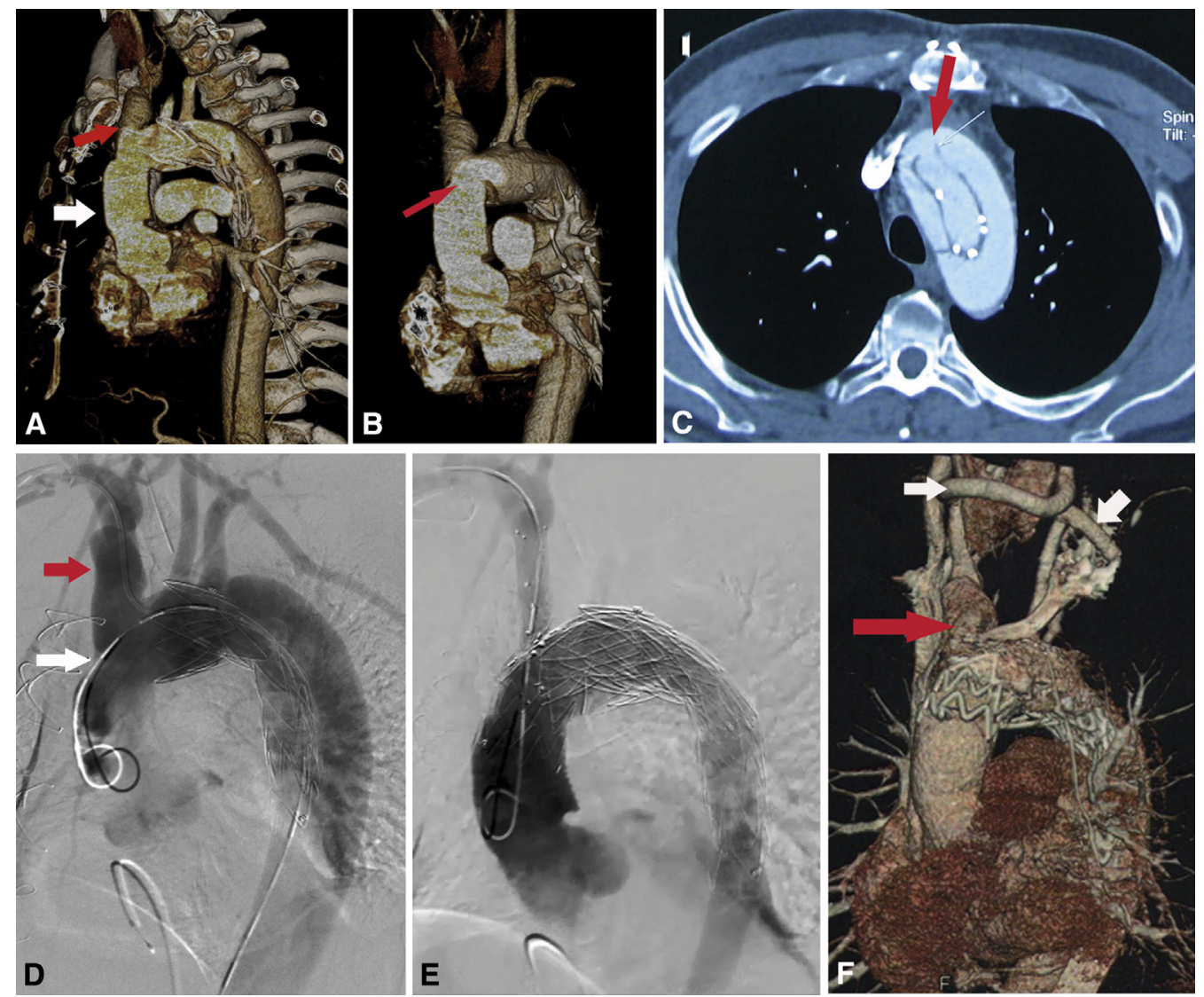

FIGURE E1. Endovascular treatment of recurrent aortic dissection after open surgery replacement of the ascending aorta. A, In patient 8 , primary endovascular repair was done to treat a Stanford type B aortic dissection. At 6 months later, new dissections occurred in the ascending aorta and were treated by open surgical replacement of the ascending aorta (white arrow). After 3 months, a new aortic dissection occurred, affecting the IA (red arrow). B, Proximal entry tear located near the distal anastomoses, in zone 0 (red arrow). C, Proximal entry tear (red arrow). The whole aortic arch was involved by the false lumen, and the true lumen was narrowed. D, Aortography showing the location of the proximal tear (white arrow) and involvement of the IA (red arrow). E, This patient was treated with a branched stent-graft. The IA was preserved by branch section. The LCA and LSA were reconstructed by cervical bypass. Final aortography showed complete exclusion of the false lumen. There was no endoleak, and the IA was patent. F, Four-year CTA confirming complete thrombosis of the false lumen of the thoracic aorta. The IA and the cervical bypasses were patent (white arrow), and the false lumen of the IA was thrombosed and shrunken. 


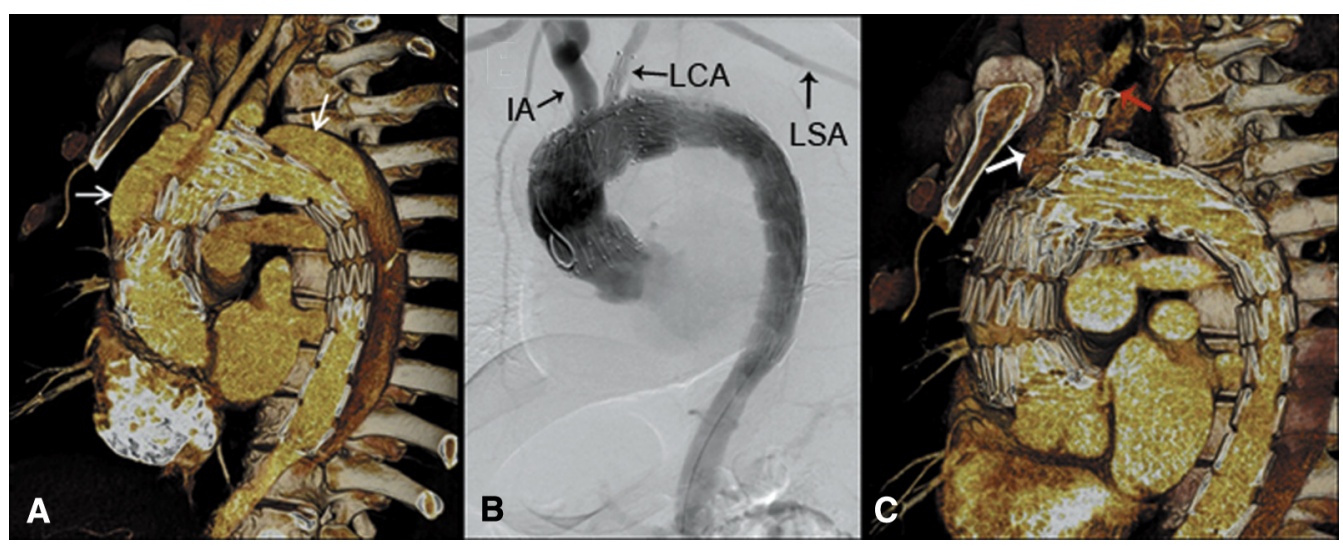

FIGURE E2. Branched stent-grafting for a new aortic dissection after a primary endovascular procedure. A, In the primary endovascular procedure for patient 12, the multiple tears in the ascending and descending aorta were excluded by endografts (42-80 and 32-170; Zenith TX2; Cook Medical, Bloomington, Ind). Shown is the 3-month follow-up CTA revealing a new dissection with an entry tear located in the arch. B, Final aortography of the secondary endovascular procedure demonstrating that the new dissection was completely excluded by the branched stent-graft with 1 fenestration. The branch section was put into the LCA, and the fenestration was aligned with the IA. The LSA was reconstructed with a bypass from the LCA. C, Four-year follow-up CTA revealing patent arch branch arteries. IA, Innominate artery, shown by the white arrow; LCA, left common carotid artery, shown by the red arrow; LSA, left subclavian artery. 

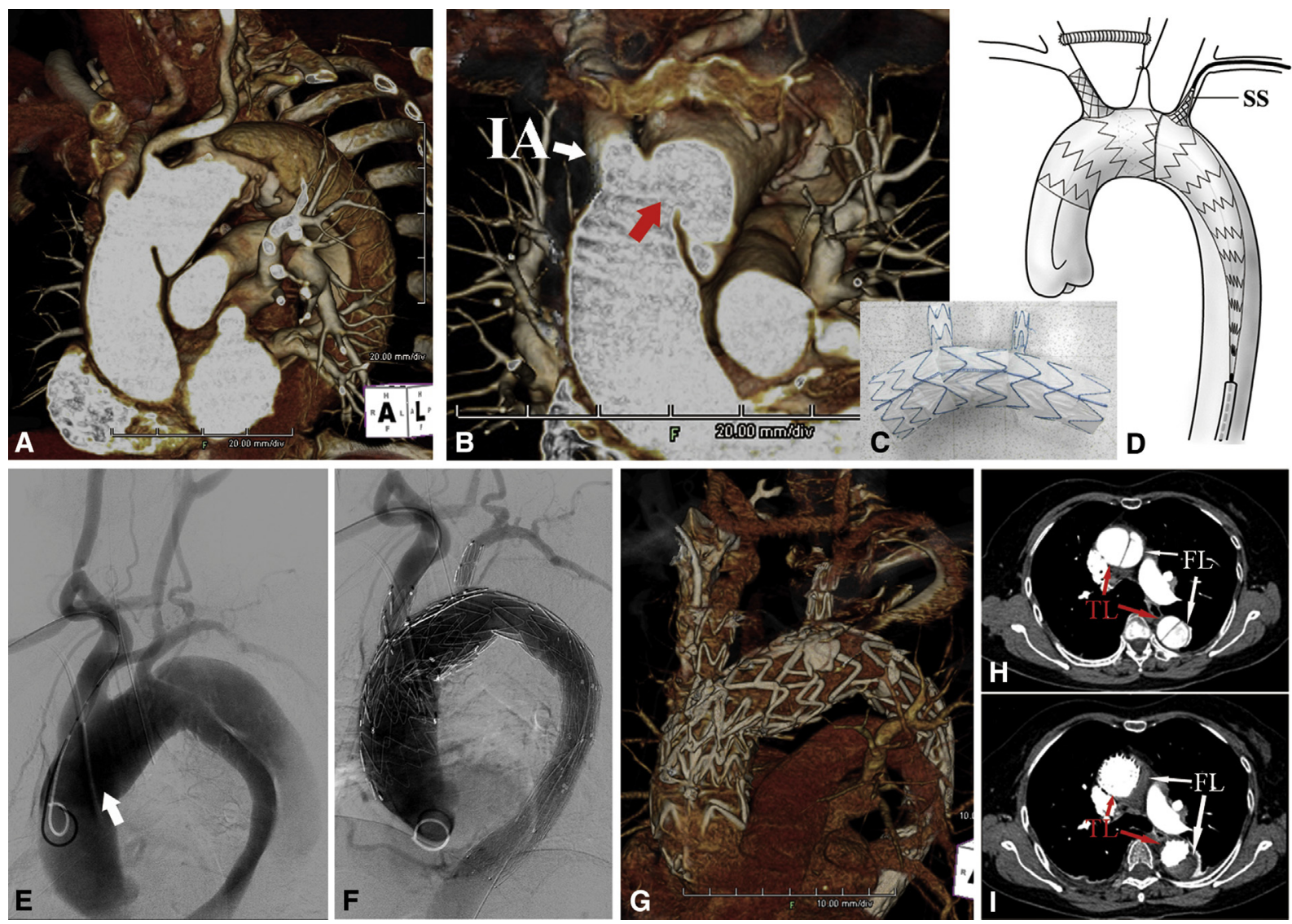

FIGURE E3. Aortic dissections with entry tear in zone 0 treated by 2 overlapping branched stent-grafts. A and B, In patient 1, preoperative CTA revealing the proximal entry tear located in zone 0 (red arrow), at the level of origin of the IA. C, In vitro view of 2 overlapping branched stent-grafts. D, Cartoon showing deployment of the second branched stent-graft. Two branch sections preserved the IA and LSA, and the LCA was reconstructed by carotid-carotid bypass. SS, soft sheath of the branch section (cap). E, Aortography showing the location of the entry tear of the aortic dissection. The cervical bypass is still patent. F, Final aortography showing complete exclusion of the false lumen and reconstruction of the arch branch arteries according to the original plan. G, Five-year CTA revealing complete thrombosis of the false lumen and patent supra-arch branches. H, Cross-sectional view of the CTA before the endovascular procedure. $T L$, true lumen; $F L$, false lumen. I, Cross-sectional view of the CTA in the follow-up period. Comparing preoperative and follow-up CTAs shows that at the same level, the false lumen is completely thrombosed and significantly shrunken, and the true lumen is expanded. $I A$, Innominate artery.

TABLE E1. Patient characteristics

\begin{tabular}{lc}
\hline \multicolumn{1}{c}{ Characteristic } & Value \\
\hline Previous aortic intervention, $\mathrm{n}$ & 4 \\
Previous open surgery & 2 (patients 8 and 13; see Figure E1) \\
Previous open surgery & 2 (patients 12 and 29; see Figure E2) \\
Localized arch dissection, $\mathrm{n}$ & 4 (patients 19, 24, 26, and 27) \\
Anatomic variants of aortic & 3 (patients 2, 18, and 31; see Table E2) \\
arch, $\mathrm{n}$ & 68 \\
Comorbidities, n & 3 \\
Cardiac dysfunction & 5 \\
Cerebrovascular lesions & 8 \\
Chronic obstructive & \\
pulmonary disease & 2 \\
Renal insufficiency & 42 \\
Hypertension & 8 \\
Diabetes mellitus & \\
\hline
\end{tabular}




\begin{tabular}{|c|c|c|c|c|c|c|}
\hline \multirow[b]{2}{*}{ No. } & \multirow[b]{2}{*}{$\begin{array}{c}\text { Proximal } \\
\text { tear location }\end{array}$} & \multirow[b]{2}{*}{ Type of stent-graft } & \multicolumn{4}{|c|}{$\begin{array}{c}\text { Strategy for supra-arch branch } \\
\text { artery reconstruction }\end{array}$} \\
\hline & & & $\begin{array}{l}\text { Branch } \\
\text { section }\end{array}$ & $\begin{array}{c}\text { First } \\
\text { fenestration }\end{array}$ & $\begin{array}{c}\text { Second } \\
\text { fenestration }\end{array}$ & $\begin{array}{c}\text { Primary cervical } \\
\text { bypass }\end{array}$ \\
\hline 1 & $\mathrm{Z} 0$ & Two single-branched & IA, LSA & - & - & $\mathrm{C}-\mathrm{C}$ \\
\hline $2 *$ & $\mathrm{Z} 2$ & Single-branched + single proximal fenestration & RSA & $\begin{array}{l}\text { Common trunk of } \\
\text { LCA and RCA } \\
\text { + LSA }\end{array}$ & - & - \\
\hline 3 & $\mathrm{Z} 2$ & Single-branched & LSA & - & - & - \\
\hline 4 & $\mathrm{Z} 2$ & Single-branched + single proximal fenestration & LSA & IA & - & $\mathrm{C}-\mathrm{C}$ \\
\hline 5 & $\mathrm{Z} 1$ & Single-branched + single proximal fenestration & LSA & IA & - & $\mathrm{C}-\mathrm{C}$ \\
\hline 6 & $\mathrm{Z} 2$ & Single-branched + single proximal fenestration & LSA & $\mathrm{IA}+\mathrm{LCA} \dagger$ & - & - \\
\hline 7 & $\mathrm{Z} 0$ & Single-branched & IA & - & - & C-C-LSA \\
\hline 8 & $\mathrm{Z} 0$ & Single-branched & IA & - & - & C-C-LSA \\
\hline 9 & $\mathrm{Z} 2$ & Single-branched + double proximal fenestrations & LSA & IA & LCA & - \\
\hline 10 & $\mathrm{Z} 2$ & Single-branched + double proximal fenestrations & LSA & IA & LCA & - \\
\hline 11 & $\mathrm{Z} 2$ & Single-branched + single proximal fenestration & LSA & $\mathrm{IA}+\mathrm{LCA}$ & - & - \\
\hline 12 & $\mathrm{Z} 1$ & Single-branched + single proximal fenestration & LCA & IA & - & LCA-LSA \\
\hline 13 & $\mathrm{Z} 0$ & Single-branched & IA & - & - & C-C-LSA \\
\hline 14 & $\mathrm{Z} 1$ & Two single-branched & IA, LSA & - & - & $\mathrm{C}-\mathrm{C}$ \\
\hline 15 & $\mathrm{Z} 2$ & Single-branched + single proximal fenestration & LSA & $\mathrm{IA}+\mathrm{LCA}$ & - & - \\
\hline 16 & $\mathrm{Z3}$ & Single-branched + single proximal fenestration & LSA & $\mathrm{IA}+\mathrm{LCA}$ & - & - \\
\hline 17 & $\mathrm{Z3}$ & Single-branched + single proximal fenestration & LSA & $\mathrm{IA}+\mathrm{LCA}$ & - & - \\
\hline $18 \ddagger$ & $\mathrm{Z3}$ & Single-branched + double proximal fenestrations & RSA & $\mathrm{LCA}+\mathrm{LSA}$ & RCA & - \\
\hline 19 & $\mathrm{Z} 1$ & Single-branched +1 proximal and 1 distal fenestration & LCA & IA & LSA & - \\
\hline 20 & $\mathrm{Z} 2$ & Single-branched + single proximal fenestration & LSA & $\mathrm{IA}+\mathrm{LCA}$ & - & - \\
\hline 21 & $\mathrm{Z3}$ & Single-branched + single proximal fenestration & LSA & $\mathrm{IA}+\mathrm{LCA}$ & - & - \\
\hline 22 & $\mathrm{Z3}$ & Single-branched + single proximal fenestration & LSA & $\mathrm{IA}+\mathrm{LCA}$ & - & - \\
\hline 23 & $\mathrm{Z} 2$ & Single-branched + single proximal fenestration & LSA & $\mathrm{IA}+\mathrm{LCA}$ & - & - \\
\hline 24 & $\mathrm{Z} 1$ & Single-branched + single proximal fenestration & LCA & IA & - & LCA-LSA \\
\hline 25 & $\mathrm{Z} 0$ & Single-branched + double distal fenestrations & IA & LCA & LSA & - \\
\hline 26 & $\mathrm{Z} 2$ & Single-branched + single proximal fenestration & LSA & $\mathrm{IA}+\mathrm{LCA}$ & - & - \\
\hline 27 & $\mathrm{Z} 1$ & Single-branched + single proximal fenestration & LSA & $\mathrm{IA}+\mathrm{LCA}$ & - & - \\
\hline 28 & $\mathrm{Z3}$ & Single-branched + double proximal fenestrations & LSA & IA & LCA & - \\
\hline 29 & $\mathrm{Z} 0$ & Single-branched + double distal fenestrations & IA & LCA & LSA & - \\
\hline 30 & $\mathrm{Z} 0$ & Single-branched + double distal fenestrations & IA & LCA & LSA & - \\
\hline $31 ब$ & $\mathrm{Z} 2$ & Single-branched +1 proximal and 1 distal fenestrations & LSA & $\mathrm{RCA}+\mathrm{LCA}$ & RSA & - \\
\hline 32 & $\mathrm{Z} 2$ & Single-branched + single proximal fenestrations & LCA & IA & - & LCA-LSA \\
\hline 33 & $\mathrm{Z3}$ & Single-branched & LSA & - & - & - \\
\hline 34 & $\mathrm{Z} 2$ & Single-branched & IA & - & - & C-C-LSA \\
\hline 35 & $\mathrm{Z} 2$ & Single-branched + double proximal fenestrations & LSA & IA & LCA & - \\
\hline 36 & $\mathrm{Z} 3$ & Single-branched & LSA & - & - & - \\
\hline 37 & $\mathrm{Z} 3$ & Single-branched & LSA & - & - & - \\
\hline 38 & $\mathrm{Z} 3$ & Single-branched & LSA & - & - & - \\
\hline 39 & $\mathrm{Z} 3$ & Single-branched & LSA & - & - & - \\
\hline 40 & $\mathrm{Z} 3$ & Single-branched & LSA & - & - & - \\
\hline 41 & $\mathrm{Z} 3$ & Single-branched & LSA & - & - & - \\
\hline 42 & $\mathrm{Z} 3$ & Single-branched & LSA & - & - & - \\
\hline 43 & $\mathrm{Z} 3$ & Single-branched & LSA & - & - & - \\
\hline 44 & $\mathrm{Z3}$ & Single-branched & LSA & - & - & - \\
\hline 45 & $\mathrm{Z} 3$ & Single-branched & LSA & - & - & - \\
\hline 46 & $\mathrm{Z3}$ & Single-branched & LSA & - & - & - \\
\hline 47 & $\mathrm{Z3}$ & Single-branched & LSA & - & - & - \\
\hline 48 & $\mathrm{Z3}$ & Single-branched & LSA & - & - & - \\
\hline
\end{tabular}


TABLE E2. Continued

Strategy for supra-arch branch

artery reconstruction

\begin{tabular}{lclcccc} 
Noximal & Proxation & Type of stent-graft & $\begin{array}{c}\text { Branch } \\
\text { section }\end{array}$ & $\begin{array}{c}\text { First } \\
\text { fenestration }\end{array}$ & $\begin{array}{c}\text { Second } \\
\text { fenestration }\end{array}$ & $\begin{array}{c}\text { Primary cervical } \\
\text { bypass }\end{array}$ \\
\hline 49 & Z3 & Single-branched & LSA & - & - & - \\
50 & Z3 & Single-branched & LSA & - & - & - \\
51 & Z3 & Single-branched & LSA & - & - \\
\hline
\end{tabular}

$C$ - $C$, Right common carotid artery-left common carotid artery bypass; $C$ - $C$ - $L S A$, right common carotid artery-left common carotid artery-left subclavian artery bypass; $L C A$ $L S A$, left common carotid artery-left subclavian artery bypass; $R S A$, right subclavian artery; $I A$, innominate artery. *In patient 2 , the supra-arch branches were aberrant; the RSA arose directly from the arch, distal to the origin of the LSA, and the LCA and RCA shared a common trunk arising from the arch, proximal to the origin of the LSA. The common trunk of the LCA and RCA, and the LSA were together preserved by one fenestration, and the RSA was preserved by a branch section of the stent-graft. †The IA and LCA connected by + means that 1 fenestration aligned with the 2 origins of the IA and LCA. ‡Patient 18 had a type III aortic arch, and a sharp angle of conjunction of part of the arch and the descending aorta. The arch branch arteries were aberrant. The LSA and LCA shared the same trunk arising from the proximal aorta. The second branch artery was the RCA, and the third branch artery was the RSA. For this special case, the branch section of the stent-graft was put into the RSA, 1 fenestration was aligned with the common trunk of the LSA and LCA, and the other was fenestration aligned with the RCA. Then the first fenestration was stented. 9 In patient 31 , the arch branch arteries were aberrant. The RSA arose directly from the arch, distal to the origin of the LSA, and the RCA shared the same origin with the LCA. In this case, the branch section of the stent-graft was deployed into the LSA, 1 fenestration was aligned with the common origin of RCA and LCA, and the other fenestration was aligned with the origin of the RSA.

TABLE E3. Thirty-day results

\begin{tabular}{lc}
\hline \multicolumn{1}{c}{ Variable } & Value \\
\hline Arch branch artery patency, \% & 100 \\
Type I/III endoleak, $\mathrm{n}$ & 0 \\
Intensive care unit stay, d & 0 \\
Stroke, $\mathrm{n}$ & 0 \\
Mortality, $\mathrm{n}(\%)$ & $1(2.0)$ \\
\hline
\end{tabular}

